\title{
Hydroxyapatite - antibiotic applications in bone therapy
}

\author{
R Mecu ${ }^{1}$, D Cîrţînă ${ }^{1}$, V Nănescu ${ }^{1}$ \\ 1“Constantin Brâncusi” University of Targu-Jiu, Faculty of Medical and Behavioral Sciences, \\ No. 4, Tineretului St., Tg-Jiu, Romania \\ E-mail: danielacirtina@gmail.com
}

\begin{abstract}
The aim of the current study is to prevent and reduce the number of bacterial infections in surgical implantation procedures. The hydroxyapatite is a calcium phosphate ceramic with important applications in the medicine and chemistry fields. The hydroxyapatite is a main mineral constituent of the hard tissues such as bones and teeth. The hydroxyapatite has remarkable properties including biocompatibility, bioactivity and ability to form a direct chemical bond with human hard tissues. Over the last decades, the biomedical orthopaedic and dentistry sectors have witnessed an unprecedented demand for a large variety and number of scaffolds, grafts, implants, and endo-prostheses. The increase in life expectancy, and the higher frequency of injuries and diseases are regarded as the main factors for this growing demand in orthopaedic and dental devices. The quality of life for millions of people has been drastically improved by using hydroxyapatite for bone repair and tissue regeneration. Recent findings in the realm of ion-substituted hydroxyapatite (HA) could pave the road towards significant developments in biomedicine, with an emphasis on a new generation of orthopaedic and dentistry applications, since such bioceramics are able to mimic the structural, compositional and mechanical properties of the bone mineral phase.
\end{abstract}

Keywords: hydroxyapatite-antibiotic, nanocomposite, physicochemical characterization

\section{Introduction}

Currently, there is an increased interest in tissue engineering and regenerative therapies, given the increased number of patients with different trauma or musculoskeletal disorders, correlated with increased life expectancy. Moreover, the need to speed up the process of healing bone fractures and to treat the various fractures has led to the consideration of a variety of drugs that determine the regeneration of bone tissue, in combination with tissue engineering; these include bisphosphonates. Reconstructive surgery is presently struggling with the problem of infections located within implantation biomaterials. Of course, the best antibacterial protection is antibiotic therapy. However, oral antibiotic therapy is sometimes ineffective, while administering an antibiotic at the location of infection is often associated with an unfavourable ratio of dosage efficiency and toxic effect [1].

Orthopedic surgery and trauma can cause infections. These complications occur only in 5\% of cases, but the treatment is complicated due to the formation of the bacterial biofilm and the limited access of the drug to the level of the infected area in case of its systemic administration. Chronic osteomyelitis is a chronic inflammatory disorder, induced by a bone infection. This can affect a single portion of the bone or multiple areas such as bone marrow, compact bone tissue, periosteum or other adjacent tissues. Despite the new medical or surgical discoveries, this has remained a challenge as it 
can persist for weeks, months or even years, requiring extended and complex management in terms of the nature of the surgery and the treatment with antibiotics needed. The standard surgical procedure involves removal of infected bone and soft tissue, a procedure described in the literature as "debridement", followed by antibiotic-based therapy administered systemically, orally or intravenously over an extended period (at least 4 or 6 weeks) with the possibility adverse effects and also the need to extend the length of hospitalization. Thus, the local treatment of bone infections became intensely studied. Reducing infections associated with implants plays an essential role in orthopedic surgery, as more and more people require implants (up to 200,000 per year in the United States (source: Joint Implant Surgery \& Research Foundation 2010). The aim of the current study is to prevent and reduce the number of bacterial infections. Both pre- and postoperative systemic antibiotic treatment and amoxicillin containing bone cement (polymethacrylate, PMMA) are strategies commonly used to overcome infections. Infections remain a critical issue in total joint arthroplasty. The addition of antibiotics to bone cement has been shown to significantly improve antimicrobial prophylaxis in cemented joint arthroplasty. Prophylaxis comparable to local antibiotics was not yet possible in joint arthroplasty without cement. The purpose of the current study is to investigate the antimicrobial effect of two amoxicillin-hydroxyapatite (HA) coatings for cementless prostheses in mice [2-4].

\section{Methods of analysis}

Hydroxyapatite can be used as a coating for metal prostheses. Metal endoprosthetics not coated with Hydroxyapatite have good mechanical strength, but are low in osteoconductive and non-absorbable.

It is found both in the natural state (bones, teeth), but it can also be obtained synthetically (physical and mechanical properties alike).

Hydroxyapatite - $\mathrm{Ca}_{10}\left(\mathrm{PO}_{4}\right)_{6}(\mathrm{OH})_{2}$ is part of the calcium phosphate family and is a major constituent of human bones and teeth. Physical Properties: Pure hydroxyapatite is a white substance; natural apatites can have different shades: brown, yellow or green. Fine salt; at high temperatures (>900 degrees Celsius) it can take the crystalline form [5,6].

The pure hydroxyapatite powder was prepared by mixing appropriate amounts of $\mathrm{Ca}(\mathrm{OH})_{2}(0.1 \mathrm{M})$ and $\mathrm{H}_{3} \mathrm{PO}_{4}(0.1 \mathrm{M})$ aqueous solutions to achieve predetermined $\mathrm{Ca} / \mathrm{P}$ atomic ratio of 1.67 [10]. The suspension obtained was aged for $3 \mathrm{~h}$ and then filtered and washed with ethanol and triply distilled water. The obtained powder was calcined $1 \mathrm{~h}$ at $800{ }^{\circ} \mathrm{C}$ in an electrically heated furnace in order to increase its crystallinity.

Due to its resemblance to natural bone tissues, synthetic hydroxyapatite has already been widely used for various applications, such as implant material or material for coating metal implants. Besides, the bismuth is one of the most investigated elements, being regarded as "the wonder metal" because of its diverse oxidation states. Its behaviour as smart optically active centres in diverse materials has been noticed in the last decades [7-9]. Due to these properties the bismuth compounds have important applications in areas of telecommunication, biomedicine, white light illumination and lasers. Webster et al. reported that the $\mathrm{Bi}^{3+}$ would be the best choice of dopant to enhance properties of HA pertinent for bone implant applications. Obtaining bismuth-substituted hydroxyapatite: $100 \mathrm{ml}$ of aqueous solution containing $\mathrm{Bi}\left(\mathrm{NO}_{3}\right)_{3} 5 \mathrm{H}_{2} \mathrm{O}$ and $\mathrm{Ca}(\mathrm{OH})_{2}$ (with molar ratio $\mathrm{Bi} /(\mathrm{Bi}+\mathrm{Ca}) 0.01 \ldots 0.20$ ) is mixed with $100 \mathrm{ml}$ aqueous solution of $\mathrm{H}_{3} \mathrm{PO}_{4} 0.1 \mathrm{M}$ so that the molar ratio $(\mathrm{Ca}+\mathrm{Bi}) / \mathrm{P} 1.67$ is present in the solution, the $\mathrm{pH}$-value is kept constant at 10.5 with $1 \mathrm{M} \mathrm{NaOH}$, the resulting precipitate is aged at $60^{\circ} \mathrm{C}$ for 24 hours under continuous stirring, then the precipitate is separated by vacuum filtration, washed with bidistilled water, dried in an oven at $90^{\circ} \mathrm{C}$ for 2 hours and calcined in an oven at $800^{\circ} \mathrm{C}$ for one hour. Hour, finally resulting in 0 nanometric powder. Finally, according to the example of preparation, a nanostructured biomaterial of hydroxyapatite type substituted with bismuth in different proportions, with the following characteristics obtained according to the invention:

- chemical composition: $\mathrm{Ca}_{10-\mathrm{x}} \mathrm{Bi}_{\mathrm{x}}\left(\mathrm{PO}_{4}\right)_{6}(\mathrm{OH})_{2}$ where $\mathrm{x} \leq 2$;

- degree of substitution $\mathrm{Ca}^{2+} \leftrightarrow \mathrm{Bi}^{3+}: 0.1 \ldots .20 \%$ (\% mass); 
- yellow powder, intensity of ass rivers increasing with increasing bismuth concentration in substituted hydroxyapatite;

- crystalline powder, with the hexagonal crystallization system;

- granulation: $35-60 \mathrm{~nm}$;

- porosity: $40-55 \%$;

- pore size: 1.2 - $2.2 \mathrm{~nm}$;

- the material exhibits an activity of antibacterial inhibition against the baits Escherichia coli and Staphylococcus aureus, the antibacterial inhibition increasing once the concentration of bismuth in the substituted hydroxyapatite increased;

- the material is radioopac, radioopacity increasing once I increase the concentration of bismuth in substituted hydroxyapatite [8-10].

Bismuth (Bi) doped HA was found to be cytocompatible with human osteoblasts [8], but induced certain levels apoptosis of human blood monocyte. Bismuth is normally not found in the human body, but when doping HA with Bi the adherence and differentiation could be enhanced. Bi-HA possesses the ability to induce the formation well-developed bone-like apatite layers after 1 month of immersion insimulated body fluid. Bismuth doping increased the dissolution rate of HA and elicited an antibacterial effect against $S$. aureusand $E$. coli, which makes Bi-HA a pertinent candidate for bone implant applications [8,9]. Excellent mechanical properties, antimicrobial activity against various pathogens, high osteoconductivity and in vitro biocompatibility was revealed. The in vivo investigations demonstrated the osteogenic potential of Bi-HA - polyurethane composite, with the authors advocating for a proper biomimetic microenvironment for bone regeneration with excellent cytocompatibility $[5,7,10]$.

Hydroxyapatite synthesis/polyvinyl alcohol/sodium alginate/amoxicillin nanocomposite $2.5 \mathrm{~g}$ sodium alginate was added to $50 \mathrm{~mL}$ of water with mixing. Hydroxyapatite nanoparticles coated with polyvinyl alcohol were added to the solution and mixed. $2.5 \mathrm{~g}$ amoxicillin was added concomitantly with the solution and stirred for 2 hours. The sample was separated by centrifugation and dried at room temperature. The blocking efficiency of the drug was calculated spectrophotometrically at the wavelength of $231 \mathrm{~nm}(1)$ :

$$
(\%)=[(\mathrm{X}-\mathrm{Y}) / \mathrm{X}] \times 100
$$

where $\mathrm{X}$ and $\mathrm{Y}$ are the initial and final concentrations of the drug

The efficacy of drug use is calculated using a standard chart for amoxicillin. Amoxicillin is prepared at different concentrations by serial dilution method. Amoxicillin is a penicillin antibiotic that fights bacteria.

Amoxicillin is used to treat many different types of infection caused by bacteria, such as tonsillitis, bronchitis, pneumonia, gonorrhea, and infections of the ear, nose, throat, skin, or urinary tract.

A standard graph is to take the OD at $231 \mathrm{~nm}$. This standard chart is used to calculate the effectiveness of drugs

Drug release: an in vitro study

100 milligram of the sample was added in $100 \mathrm{~mL}$ of phosphate buffer saline (PBS) in a glass vial at $37^{\circ} \mathrm{C}$ at $\mathrm{pH}=7.4$. The drug release was analyzed for 30 days. The $5 \mathrm{~mL}$ sample was withdrawn at constant time intervals. The withdrawn buffer was replaced immediately with $5 \mathrm{~mL}$ of fresh PBS medium. Amoxicillin concentration in the collected samples was measured at $231 \mathrm{~nm}$ spectrophotometrically [10].

\section{Conclusions}

Hydroxyapatite plays a key role in bioceramic and biomaterial composites and is commonly used in reconstruction medicine and dental implantology.

Antibacterial activity of hydroxyapatite was analyzed against Bacillus subtilis, Klebsiella pneumoniae using the agar diffusion method. The antibacterial activity was done by the diffusion method well against Bacillus subtilis and Klebsiella pneumonia. These two organisms are important because they cause bone infections. The zone of inhibition against the two bone infections increases 
the pathogens with increases in concentration. This shows very good antibacterial activity for nanocomposites loaded with drugs. Hydroxyapatite nanoparticles will also exhibit antimicrobial effect. The inhibition zone is almost equal to the standard drug Amoxicillin at lower concentration and is higher than the standard drug at higher concentration This is due to the sustained release of drugs from nanocomposite. It is very difficult to cure bone infections, especially infections associated with implants. Antibiotics such as Amoxicillin should be taken for a long time. A sustained release of drugs will be more effective in treating bone infections. Sustained release of the drug from the nanocomposite exhibits effective antibacterial activity. The layer-by-layer polymer coating on the drug amoxicillin is highly effective in sustained release of amoxicillin. The nanocomposite was synthesized for sustained release of amoxicillin. PVA and sodium alginate are coated layer by layer on hydroxyapatite nanoparticles. Thematic layer of polymer layer results in sustained release of amoxicillin. A sustained release of the antibiotic is observed from the nanocomposite for 30 days. The nanocomposite loaded with amoxicillin showed very good antibacterial activity, compared to the standard drug. This ensures that this nanocomposite can be used as a delivery system of antibiotic for bone infections.

\section{References}

[1] Miculescu F., Maidaniuc A, Voicu S.I., Thakur V.K., Stan G.E., Ciocan L.T., Progress in hydroxyapatites based sustainable biomaterials for biomedical bone substitution applications. ACS Sustain Chem Eng., 2017.

[2] Nakanishi K, Sakiyama T, Imamura K., On the adsorption of proteins on solid surfaces, a common but very complicated phenomenon. J. Biosci. Bioeng., 2001.

[3] Palazzo B., Iafisco M., Laforgia M., Margiotta N, Natile G. et al., Biomimetic hydroxyapatite drug nanocrystals as potential bone substitutes with antitumor drug delivery properties. Adv. Funct. Mater., 2007.

[4] Brajendra S., Dubey A.K., Kumar S., Saha N., Basu B., Gupta R., In vitrobiocompatibility and antimicrobial activity, 2010.

[5] Ciobanu, C.S., Popa, C.L., Predoi, D., Cerium doped hydroxyapatite nanoparticles synthesized by coprecipitation method, J. Serb. Chem. Soc., 2016.

[6] Ciobanu, C.S., Iconaru, S.L., Le Coustumer, P., Predoi, D., Vibrational investigations of silverdoped hydroxyapatite with antibacterial properties, J. Spectrosc., 2013.

[7] Supova, M., Substituted hydroxyapatites for biomedical applications: A review. Ceram. Int., 2015.

[8] Singh, M., Singh, S., Prasad, S., Gambhir, I.S., Nanotechnology in medicine and antibacterial effect of silver nanoparticles, Dig. J. Nanomater. Biostruct., 2008.

[9] Singh S., Kumar R. M., Kuntal K. K. et al., "Sol-gel derived hydroxyapatite coating on Mg-3Zn alloy for orthopedic application," JOM: The Journal of the Minerals, Metals \& Materials Society, 2015.

[10] Sternitzke V., Janousch M., Heeb M. B., Hering J. G. and Johnson C. A., "Strontium hydroxyapatite and strontium carbonate as templates for the precipitation of calcium-phosphates in the absence and presence of fluoride," Journal of Crystal Growth, 2014. 